\title{
Hippocampus-sparing whole-brain radiotherapy: dosimetric comparison between non-coplanar and coplanar planning
}

\author{
Li-Jhen Chen ${ }^{1}$, Ming-Hsien Li ${ }^{1,2}$, Hao-Wen Cheng ${ }^{1,3}$, Chun-Yuan Kuo ${ }^{1,3}$, Wei-Lun Sun ${ }^{1}$, Jo-Ting Tsai ${ }^{1,2}$ \\ ${ }^{1}$ Department of Radiation Oncology, Shuang Ho Hospital, Taipei Medical University, Taipei, Taiwan; ${ }^{2}$ Department of Radiology, School of \\ Medicine, College of Medicine, Taipei Medical University, Taipei, Taiwan; ${ }^{3}$ School of Biomedical Engineering, College of Biomedical Engineering, \\ Taipei Medical University, Taipei, Taiwan \\ Contributions: (I) Conception and design: LJ Chen, MH Li; (II) Administrative support: JT Tsai; (III) Provision of study material or patients: JT Tsai, \\ MH Li; (IV) Collection and assembly of data: LJ Chen, CY Kuo, HW Cheng, WL Sun; (V) Data analysis and interpretation: LJ Chen, CY Kuo, \\ HW Cheng; (VI) Manuscript writing: All authors; (VII) Final approval of manuscript: All authors. \\ Correspondence to: Jo-Ting Tsai, MD, PhD. Department of Radiation Oncology, Shuang Ho Hospital, Taipei Medical University, No. 291, \\ Zhongzheng Rd., Zhonghe Dist., New Taipei City 235, Taiwan. Email: kitty4024@gmail.com.
}

Background: To compare non-coplanar and coplanar volumetric-modulated arc therapy (VMAT) for hippocampal avoidance during whole-brain radiotherapy (HA-WBRT) using the Elekta Synergy and Pinnacle treatment planning system (TPS) according to the suggested criteria of the radiation therapy oncology group (RTOG) 0933 trial.

Methods: Nine patients who underwent WBRT were selected for this retrospective study. The hippocampus was contoured, and the hippocampal avoidance regions were created using a 5-mm volumetric expansion around the hippocampus for each patient. Non-coplanar and coplanar VMAT plans were generated for each patient. All treatment plans were generated for a prescribed dose (PD) of 30 Gy in 10 fractions.

Results: The average volumes of the hippocampus and hippocampal avoidance region were $2.8 \pm 0.38$ and $27 \pm 2.48 \mathrm{~cm}^{3}$, respectively. For coplanar and non-coplanar VMAT plans, the average $\mathrm{D}_{100 \%}$ of the hippocampus was 8.60 Gy (range, 8.30-8.80 Gy) and 8.56 Gy (range, 8.30-8.90 Gy), respectively, and the average $\mathrm{D}_{\max }$ of the hippocampus was 15.29 Gy (range, 14.35-15.92 Gy) and 14.99 Gy (range, 13.80-15.83 Gy), respectively. The non-coplanar VMAT plans showed a significantly lower average $\mathrm{D}_{\max }$ of the lens (4.23 Gy) than did the coplanar VMAT plans (4.77 Gy). The average gamma passing rate for non-coplanar and coplanar VMAT quality assurance (QA) with criteria of $3 \% / 3 \mathrm{~mm}$ were $95.4 \% \pm 2.6 \%$ and $95.6 \% \pm 1.6 \%$, indicating good agreement between the calculated plan dose and the measured dose.

Conclusions: We showed that the suggested criteria of the RTOG 0933 trial for the hippocampal dose can be achieved in both coplanar and non-planar VMAT plans. We performed VMAT QA for each treatment plan to verify the clinical feasibility.

Keywords: Hippocampus; RTOG 0933; radiation therapy; volumetric-modulated arc therapy (VMAT); whole brain

Received: 10 July 2020; Accepted: 25 January 2021; Published: 30 March 2021.

doi: $10.21037 /$ tro-20-50

View this article at: http://dx.doi.org/10.21037/tro-20-50

\section{Introduction}

Brain metastases are the most common intracranial tumors in adults and occur in $10-30 \%$ of cancer patients (1). The prognosis of patients with brain metastases is generally poor, with a median survival time of one month in untreated patients and 4-6 months in treated patients (2). Many cancer patients will eventually develop brain metastases, and the standard therapeutic approaches for patients with brain metastasis include surgery and radiotherapy. Whole-brain radiotherapy (WBRT) has been implemented in clinical practice for decades. In general, patients with oligo-brain 
metastasis undergo surgical resection, chemotherapy and stereotactic radiosurgery (SRS) alone or SRS combined with WBRT (3). For patients with multiple brain metastases, WBRT is a commonly used treatment modality; however, SRS seems to be a new standard option (4).

WBRT plays an important role in tumor control for patients with multiple brain tumors and thus lower the subsequent neurological death. WBRT is also used in prophylactic cranial irradiation for patients with small cell lung carcinoma (5-7). Although WBRT was proven to be effective in eliminating brain tumors, damage to normal brain tissue is inevitable, and several complications have been observed following treatment that decreases the quality of life of patients (8). Previous studies showed that poor neurocognitive function may occur months after radiotherapy due to radiation-induced hippocampal damage. The radiation-induced hippocampal damage causes a decline in neurocognitive function, including deficiencies in learning, memory and spatial management, which can be observed in patients. The clinical symptoms of radiationinduced hippocampal damage include memory loss, lack of concentration, and emotional disorders $(9,10)$.

The findings of radiation therapy oncology group (RTOG) 0933 trial suggest that hippocampal avoidance during WBRT (HA-WBRT) could reduce radiationinduced neurocognitive toxicities (11). The purpose of HAWBRT is to generate hippocampal avoidance regions while allowing the sufficient prescribed dose (PD) to cover the remainder of the brain. HA-WBRT can significantly reduce the hippocampal dose. Although the risk of developing subsequent brain metastasis after HA-WBRT is unknown, Ghia et al. (12) reported that of 272 cases with brain metastases, the incidence of metastases within $5 \mathrm{~mm}$ of the perihippocampal region was low (3.3\%). Thus, their results indicated that a $5-\mathrm{mm}$ margin around the hippocampus for HA-WBRT was an acceptable risk. With advances in radiotherapy, several reports have discussed the application of intensity-modulated radiotherapy (IMRT) and volumetric-modulated arc therapy (VMAT) for HA-WBRT and attempted to minimize WBRT-induced neurocognitive toxicities. Nevelsky et al. (13) evaluated the feasibility of the RTOG 0933 trial using the Elekta Infinity (Elekta AB, Stockholm, Sweden) equipped with Monaco 3.1 (Elekta AB, Stockholm, Sweden) treatment planning system (TPS) to perform IMRT with seven coplanar plus two non-coplanar beams. VMAT, with continuous gantry rotation and leaf motion with a varying dose rate, can increase treatment efficiency and reduce the amount of patient motion during the treatment (14). Therefore, the purpose of this study is to compare the non-coplanar and coplanar VMAT for HAWBRT using the Elekta Synergy (Elekta AB) and Pinnacle (Philips Medical System, Fitchburg, WI, USA) TPS according to the suggested criteria of the RTOG 0933 trial. We also performed VMAT quality assurance (QA) for each treatment plan to evaluate the feasibility of HA-WBRT with different VMAT techniques.

We present the following article in accordance with the MDAR reporting checklist (available at http://dx.doi. org/10.21037/tro-20-50).

\section{Methods}

\section{Patient selection and delineation}

The study was approved by the Taipei Medical University Joint Institutional Review Board, and the IRB number is TMU-JIRB No. N201711047. Nine patients with brain metastases outside $5 \mathrm{~mm}$ of the hippocampus who underwent WBRT were selected for this retrospective study. Computed tomography (CT) and magnetic resonance (MR) images were used for each patient to delineate the hippocampus, hippocampal avoidance regions (defined as the hippocampus with an expansion of $5-\mathrm{mm}$ margin) according to the RTOG 0933 trial, optic nerves, optic chiasm, and lens. CT images (3 mm slice thickness) for treatment planning were generated using a Phillips 16 slice CT scanner (Brilliance CT Big Bore). All 6-MV VMAT plans were optimized with Pinnacle ${ }^{3}$ Version 14 for Elekta Synergy with 40 multileaf collimator (MLC) leaf pairs (MLCi) of $1 \mathrm{~cm}$ leaf width at isocenter.

The study was conducted in accordance with the Declaration of Helsinki (as revised in 2013). The study was approved by the Taipei Medical University Joint Institutional Review Board (TMU-JIRB No. N201711047), and informed consent was taken from all the patients.

\section{Planning technique}

According to the RTOG 0933 trial, the clinical target volume (CTV) was defined as the whole brain parenchyma to $\mathrm{C} 1$, and the planning treatment volume (PTV) was defined as the CTV excluding the hippocampal avoidance regions in this study. A total dose of 30 Gy in 10 fractions was prescribed to the PTV. All plans in this study aimed to cover at least $90 \%$ of the PTV by the PD. The following dose constraints, which are summarized in Table 1, were 
Table 1 RTOG 0933 compliance criteria and critical structure constraint

\begin{tabular}{lccc}
\hline Parameter & Per protocol & $\begin{array}{c}\text { Variation } \\
\text { acceptable }\end{array}$ & $\begin{array}{c}\text { Deviation } \\
\text { unacceptable }\end{array}$ \\
\hline PTV & $\mathrm{D}_{2 \%} \leq 37.5 \mathrm{~Gy}$ & $\begin{array}{c}\mathrm{D}_{2 \%}>37.5 \mathrm{~Gy}, \\
\leq 40 \mathrm{~Gy}\end{array}$ & $\mathrm{~V}_{30 \mathrm{~Gy}}<90 \%$ \\
& $\mathrm{D}_{98 \%} \geq 25 \mathrm{~Gy}$ & $\begin{array}{c}\mathrm{D}_{98}<25 \mathrm{~Gy} \\
\text { Hippocampus }\end{array}$ & $\mathrm{D}_{2 \%}>40 \mathrm{~Gy}$ \\
& $\mathrm{D}_{100 \%} \leq 9 \mathrm{~Gy}$ & $\mathrm{D}_{100 \%} \leq 10 \mathrm{~Gy}$ & $\mathrm{D}_{100 \%}>10 \mathrm{~Gy}$ \\
& $\mathrm{D}_{\max } \leq 16 \mathrm{~Gy}$ & $\mathrm{D}_{\max } \leq 17 \mathrm{~Gy}$ & $\mathrm{D}_{\max }>17 \mathrm{~Gy}$ \\
\hline
\end{tabular}

$\mathrm{D}_{2} \%$, maximum dose to $2 \%$ volume, $\mathrm{D}_{98} \%$, minimum dose to $98 \%$ volume; $D_{100 \%}$, dose to $100 \%$ volume; Dmax, maximum dose; $\mathrm{V}_{30 \mathrm{G} y}$, percent volume covered by $30 \mathrm{~Gy}$.

Table 2 Gantry, collimator, and couch angles for the coplanar and non-coplanar VMAT beam arrangements

\begin{tabular}{lccc}
\hline Beam name & $\begin{array}{c}\text { Gantry } \\
\text { (degree) }\end{array}$ & $\begin{array}{c}\text { Collimator } \\
\text { (degree) }\end{array}$ & $\begin{array}{c}\text { Couch } \\
\text { (degree) }\end{array}$ \\
\hline \multicolumn{2}{l}{ Coplanar beam arrangement } & & \\
V1 & CW181-180 & 45 & 0 \\
V2 & CCW180-181 & 45 & 0 \\
V3 & CW181-180 & 45 & 0 \\
V4 & CCW180-181 & 45 & 0 \\
Non-coplanar beam arrangement & & \\
V1 & CW181-180 & 45 & 0 \\
V2 & CCW180-181 & 45 & 0 \\
V3 & CW181-180 & 45 & 0 \\
V4 & CCW180-181 & 45 & 0 \\
V5 & CW190-310 & 330 & 70 \\
\hline
\end{tabular}

CW, clockwise; CCW, counterclockwise; VMAT, volumetricmodulated arc therapy.

used in optimization: maximum dose to $2 \%$ of the PTV $\left(\right.$ PTV D $\left._{2 \%}\right) \leq 37.5$ Gy; minimum dose to $98 \%$ of the PTV $\left(\right.$ PTV $\left.D_{98 \%}\right) \geq 25$ Gy; $D_{100 \%}\left(D_{x \%}\right.$ : the dose to $X \%$ of the volume ) and maximum dose $\left(\mathrm{D}_{\max }\right)$ of the hippocampus $\leq 9$ Gy and $\leq 16$ Gy; $D_{\max }$ of the optic chasm and optic nerve $<37.5 \mathrm{~Gy}$; and doses to the lens and optic nerve as low as possible. The non-coplanar and coplanar VMAT plans were generated for each patient. For the coplanar VMAT plans, four full arcs (FAs) (clockwise for two FAs and counterclockwise for two FAs) were used with a collimator angle of $45^{\circ}$. For the non-coplanar VMAT plans, five arcs, including one partial arc (PA) with a collimator angle of $45^{\circ}$ and couch angle of $70^{\circ}$ and four FAs (clockwise for two FAs and counterclockwise for two FAs), were used (Table 2).

\section{Plan evaluation}

For the organs at risk (OARs) and PTV, the following parameters were evaluated: PTV $\mathrm{D}_{2 \%}$, PTV $\mathrm{D}_{98 \%}, \mathrm{D}_{100 \%}$ and $\mathrm{D}_{\max }$ of the hippocampus, and $\mathrm{D}_{\max }$ of the optic chiasm and lens. The dosimetric parameters, including PTV $V_{100}$ (PTV $V_{X}$ is the percent volume of PTV covered by $\mathrm{X} \%$ of the PD), PTV $V_{95}$, homogeneity index (HI) and conformity index (CI) for the PTV, were evaluated.

The HI is defined as follows (recommended by the ICRU 83) (15):

$\mathrm{HI}=\left(\mathrm{D}_{2 \%}-\mathrm{D}_{98 \%}\right) / \mathrm{D}_{\text {median }}$

where $\mathrm{D}_{\text {median }}$ is the median dose of the PTV.

The $\mathrm{CI}$ is defined as follows (16):

$\mathrm{CI}=\left(\mathrm{PTV} \mathrm{V}_{95 \%}\right)^{2} /\left(\mathrm{V}_{\mathrm{PTV}} \times \mathrm{V}_{95}\right)$

where $V_{\text {PTV }}$ is the volume of PTV, and $V_{95}$ is the volume covered by $95 \%$ of the PD.

The beam-on time was defined as the treatment time excluding the time for patient setup and couch rotation. The beam-on times for different VMAT plans were reported and evaluated. The VMAT QA procedures were completed for each plan and performed by measuring the dose distribution using the PTV 2D-array seven29 with a plane matrix of $27 \times 27$ air-filled ion chambers (17).

\section{Statistical analysis}

The Wilcoxon signed-rank test was used for statistical analysis among different VMAT techniques. A P value of $\leq 0.05$ was considered statistically significant.

\section{Results}

The basic characteristics of the nine patients are listed in Table 3. The average volume of the hippocampus was $2.80 \mathrm{~cm}^{3}$ (range, $2.32-3.40 \mathrm{~cm}^{3}$ ), the average volume of the hippocampal avoidance region was $27.09 \mathrm{~cm}^{3}$ (range, 24.76$31.26 \mathrm{~cm}^{3}$ ), and the average volume of the whole brain was $1,314.35 \mathrm{~cm}^{3}$ (range, 1,116.01-1,479.70 $\mathrm{cm}^{3}$ ).

In all treatment plans, the dose constraints for the hippocampus according to the RTOG 0933 trial were prioritized while limiting the dose to the lens. Figure 1 shows the isodose lines (ranging from 1,500 to 3,400 cGy) around the hippocampus of the non-coplanar and coplanar 
Table 3 Basic characteristics of nine patients

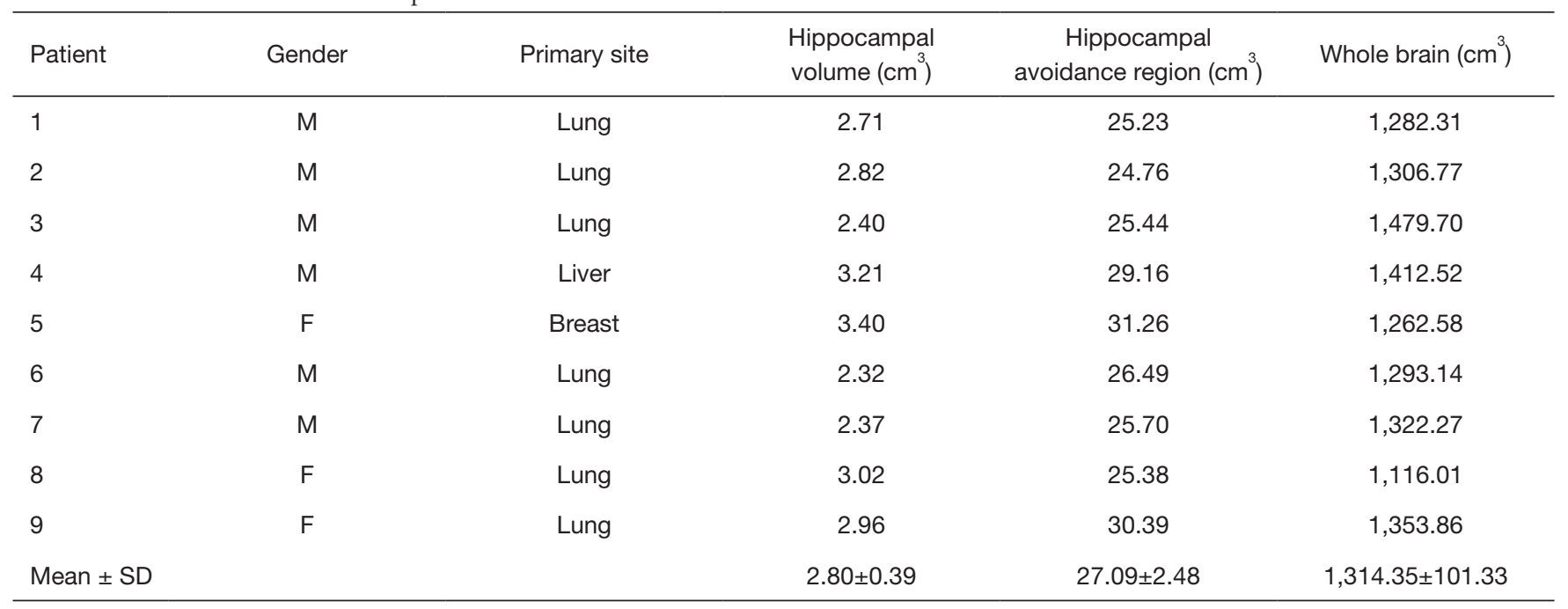

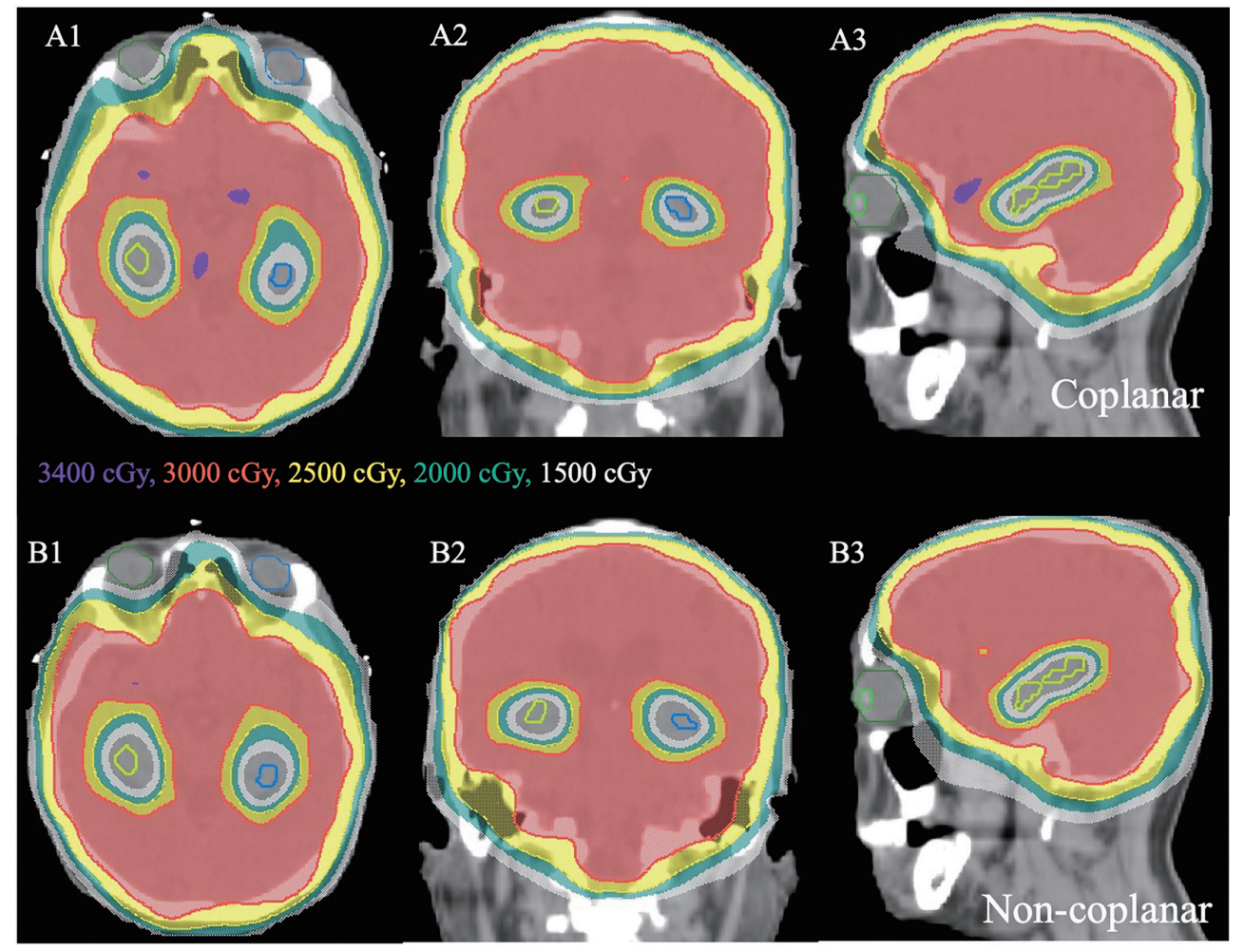

Figure 1 Isodose distributions in axial, sagittal, and coronal views for representative hippocampal sparing WBRT using coplanar VMAT (A1A3) vs. non-coplanar VMAT (B1-B3) in patient 7. Yellow-green contours indicate the right hippocampus, and light-blue contours indicate the left hippocampus. WBRT, whole-brain radiotherapy; VMAT, volumetric-modulated arc therapy. 


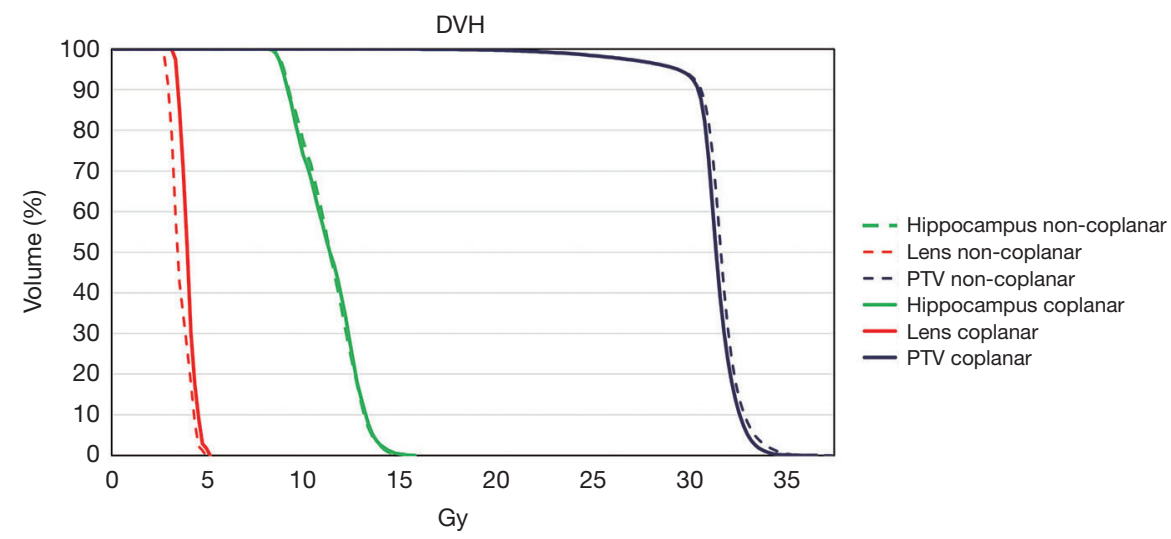

Figure 2 Comparison of the DVHs between the coplanar VMAT and non-coplanar VMAT plans. DVH, dose volume histograms; VMAT, volumetric-modulated arc therapy; PTV, planning treatment volume.

Table 4 Comparison of dosimetric parameters for OARs with $\mathrm{P}$ values

\begin{tabular}{|c|c|c|c|c|c|c|c|c|}
\hline \multirow{3}{*}{ Patient } & \multicolumn{4}{|c|}{ Hippocampus } & \multirow{2}{*}{\multicolumn{2}{|c|}{ Lens, $D_{\max }(G y)$}} & \multirow{2}{*}{\multicolumn{2}{|c|}{ Optic chiasm, D $\max (G y)$}} \\
\hline & \multicolumn{2}{|c|}{$D_{\max }$ (Gy) } & \multicolumn{2}{|c|}{$D_{\min }(\mathrm{Gy})$} & & & & \\
\hline & Coplanar & Non-coplanar & Coplanar & Non-coplanar & Coplanar & Non-coplanar & Coplanar & Non-coplanar \\
\hline 1 & 14.70 & 15.22 & 8.80 & 8.90 & 4.72 & 3.72 & 32.65 & 32.77 \\
\hline 2 & 15.34 & 14.43 & 8.54 & 8.33 & 3.99 & 4.36 & 34.72 & 34.08 \\
\hline 4 & 15.68 & 15.28 & 8.69 & 8.36 & 4.49 & 4.07 & 33.54 & 34.26 \\
\hline 5 & 15.92 & 15.22 & 8.61 & 8.30 & 5.32 & 4.62 & 33.91 & 34.18 \\
\hline 6 & 14.35 & 14.05 & 8.30 & 8.88 & 4.64 & 4.24 & 35.04 & 35.67 \\
\hline 7 & 14.99 & 13.80 & 8.51 & 8.53 & 4.56 & 3.68 & 32.64 & 33.15 \\
\hline Mean \pm SD & $15.29 \pm 0.52$ & $14.99 \pm 0.73$ & $8.60 \pm 0.18$ & $8.56 \pm 0.23$ & $4.77 \pm 0.45$ & $4.23 \pm 0.5$ & $33.58 \pm 0.87$ & $33.94 \pm 1.00$ \\
\hline$P$ value & \multicolumn{2}{|c|}{0.173} & \multicolumn{2}{|c|}{0.953} & \multicolumn{2}{|c|}{$0.015^{\star}$} & \multicolumn{2}{|c|}{0.066} \\
\hline
\end{tabular}

*, $\mathrm{P}<0.05$.

VMAT plans on axial, coronal, and sagittal images from one case of this study. The average DVHs of the hippocampus, lens and PTV for the non-coplanar and coplanar VMAT plans for nine patients are shown in Figure 2.

\section{Dose to OARs}

Table 4 shows the dose to the OARs for the non-coplanar and coplanar VMAT plans. The non-coplanar VMAT plans had a lower mean $\mathrm{D}_{\max }$ of the hippocampus than the coplanar VMAT plans, but the difference was nonsignificant $(\mathrm{P}>0.05)$ : coplanar VMAT $=15.29$ Gy (range, $14.35-$ $15.92 \mathrm{~Gy}$ ) and non-coplanar VMAT $=14.99 \mathrm{~Gy}$ (range, $13.80-15.83 \mathrm{~Gy})$. The average $\mathrm{D}_{100 \%}$ values of the hippocampus for the coplanar and non-coplanar VMAT plans were 8.60 Gy (range, 8.30-8.80 Gy) and 8.56 Gy (range, 8.30-8.90 Gy), respectively. The non-coplanar VMAT plans showed a significantly lower average $D_{\text {max }}$ of the lens $(\mathrm{P}<0.05)$ than the coplanar VMAT plans: coplanar $\mathrm{VMAT}=4.77$ Gy (range, 3.99-5.50 Gy) and non-coplanar 


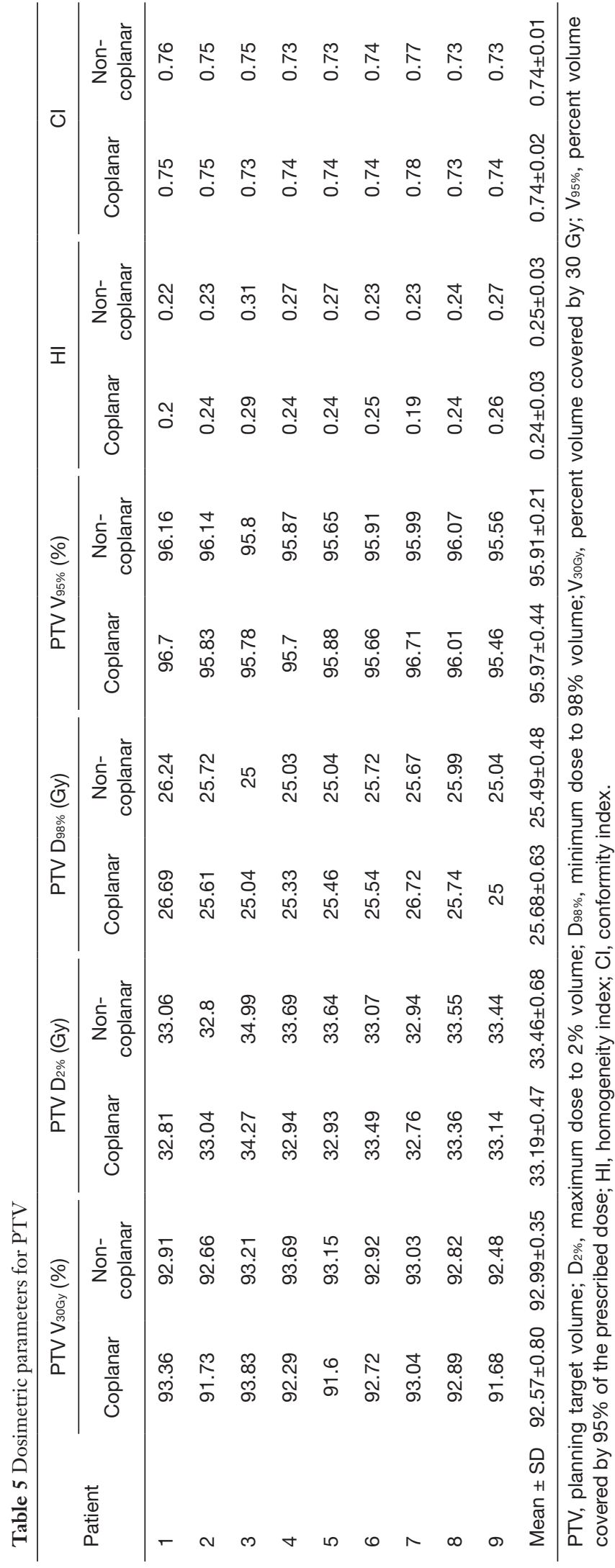

VMAT $=4.23$ Gy (range, 3.65-5.07 Gy). The dose to the optic chiasm for all plans did not exceed $37.5 \mathrm{~Gy}$.

\section{PTV dosimetry}

The PTV $\mathrm{D}_{2 \%}$, PTV $\mathrm{D}_{98 \%}$, PTV $\mathrm{V}_{30 \mathrm{~Gy}}$ (the percent volume of PTV covered by 30 Gy), PTV $\mathrm{V}_{95}$, target coverage, $\mathrm{HI}$ and CI are shown in Table 5. The average PTV $\mathrm{D}_{2 \%}$, PTV $\mathrm{D}_{98 \%}$, and PTV $\mathrm{V}_{30 \mathrm{~Gy}}$ for the non-coplanar and coplanar VMAT plans were $33.46 \pm 0.68$ and $33.19 \pm 0.47 \mathrm{~Gy}$, $25.49 \pm 0.48$ and $25.68 \pm 0.63 \mathrm{~Gy}$, and $92.99 \% \pm 0.35 \%$ and $92.57 \% \pm 0.80 \%$, respectively.

\section{Beam-on time and VMAT QA}

The beam-on time and VMAT QA results for nine patients are shown in Table 6. There was no significant difference $(\mathrm{P}>0.05)$ in the beam-on time between the non-coplanar (average, $6.2 \pm 1.3 \mathrm{~min}$ ) and coplanar VMAT plans (average, $5.6 \pm 0.5 \mathrm{~min})$. The results of the VMAT QA are shown in Figure 3. The average gamma passing rates for the noncoplanar and coplanar VMAT QA with criteria of $3 \% / 3 \mathrm{~mm}$ were $95.4 \% \pm 2.6 \%$ and $95.6 \% \pm 1.6 \%$, respectively, which indicated good agreement between the calculated plan dose and the measured dose.

\section{Discussion}

Nevelsky et al. (13) evaluated the feasibility of the RTOG 0933 trial using the Elekta Infinity to perform IMRT with seven coplanar plus two non-coplanar beams. We hypothesized that VMAT could achieve the same results. In this study, we performed HA-WBRT using non-coplanar and coplanar VMAT with Elekta Synergy. Our results showed that for all VMAT plans, the hippocampal dose can achieve the suggested criteria of the RTOG 0933 trial, with $\mathrm{D}_{\max }$ of the PTV (PTV $\mathrm{D}_{\max }$ ) lower than $37.5 \mathrm{~Gy}$, while maintaining the PTV coverage (at least $90 \%$ of the PTV by the PD).

In comparison with the study of Nevelsky et al. (13), their results showed that the average PTV $\mathrm{D}_{98 \%}$ was 25.7 Gy, PTV $\mathrm{D}_{2 \%}$ was $37.2 \mathrm{~Gy}$, HI was 0.36 , and $\mathrm{D}_{\max }$ of the hippocampus was $14.3 \mathrm{~Gy}$, and our results showed that the average $\mathrm{D}_{\max }$ of the hippocampus was slightly higher (14.9 Gy); however, our results showed better average PTV $\mathrm{D}_{2 \%}$ (33.46 Gy), PTV D $98 \%$ (25.49 Gy), and HI (0.25).

Gondi et al. (18) proposed 9-beam non-planar IMRT with nine different couch angles for HA-WBRT, and their 
Table 6 Gamma passing rate values (\%) with different criteria $(3 \% / 3 \mathrm{~mm}, 2 \% / 2 \mathrm{~mm})$ and beam-on time

\begin{tabular}{|c|c|c|c|c|c|c|}
\hline Patient & \multicolumn{2}{|c|}{$3 \% / 3 \mathrm{~mm}$} & \multicolumn{2}{|c|}{$2 \% / 2 \mathrm{~mm}$} & \multicolumn{2}{|c|}{ Beam-on time (min) } \\
\hline 1 & 96.2 & 98.0 & 88.1 & 91.2 & 5.8 & 5.7 \\
\hline 2 & 94.7 & 95.1 & 86.7 & 88.0 & 5.1 & 5.7 \\
\hline 3 & 95.5 & 95.5 & 89.3 & 88.8 & 5.1 & 9.8 \\
\hline 5 & 92.8 & 91.4 & 83.7 & 82.1 & 6.3 & 5.9 \\
\hline 6 & 96.9 & 92.5 & 87.6 & 86.1 & 5.0 & 5.7 \\
\hline 7 & 93.9 & 98.1 & 86.0 & 92.7 & 6.3 & 5.5 \\
\hline 8 & 97.7 & 97.5 & 90.0 & 90.0 & 5.0 & 5.5 \\
\hline
\end{tabular}

A Coplanar

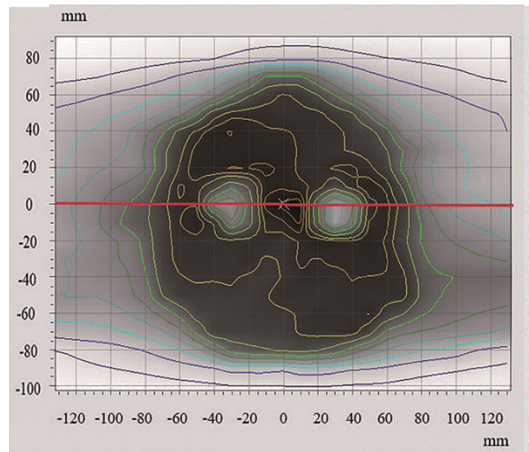

B Non-coplanar

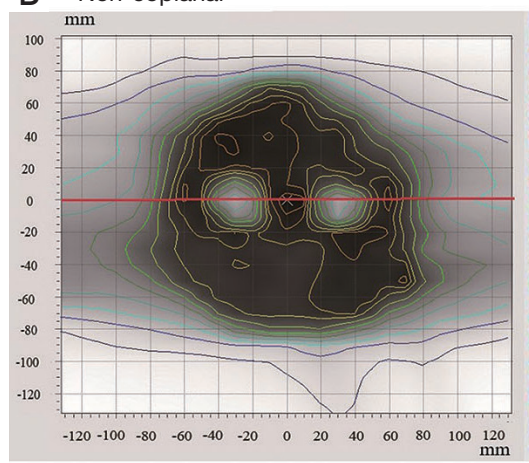

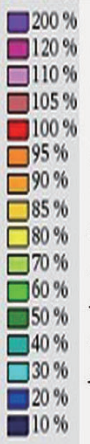
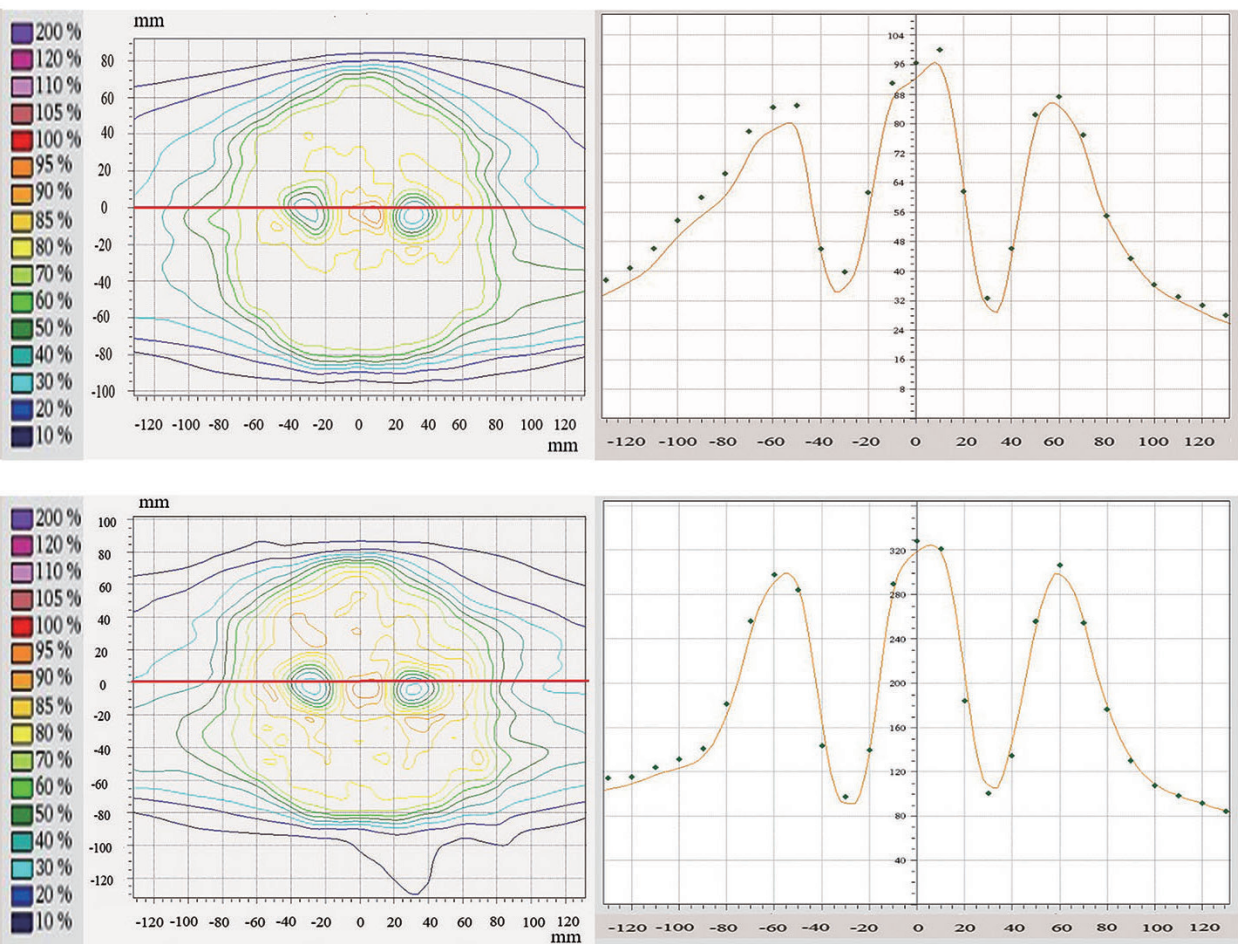

Figure 3 Calculated and measured dose profiles. (A) A coplanar VMAT plan vs. (B) a non-coplanar VMAT plan for patient 7. Left: measured dose distributions. Middle: calculated dose distribution. Lower graph: comparison of the measured dose and calculated dose (solid line). The gamma passing rates $(3 \% / 3 \mathrm{~mm})$ for the coplanar and non-coplanar VMAT plans were $93.9 \%$ and $98.1 \%$, respectively. VMAT, volumetricmodulated arc therapy. 
Table 7 Summary of dosimetric results for HA-WBRT (mean value)

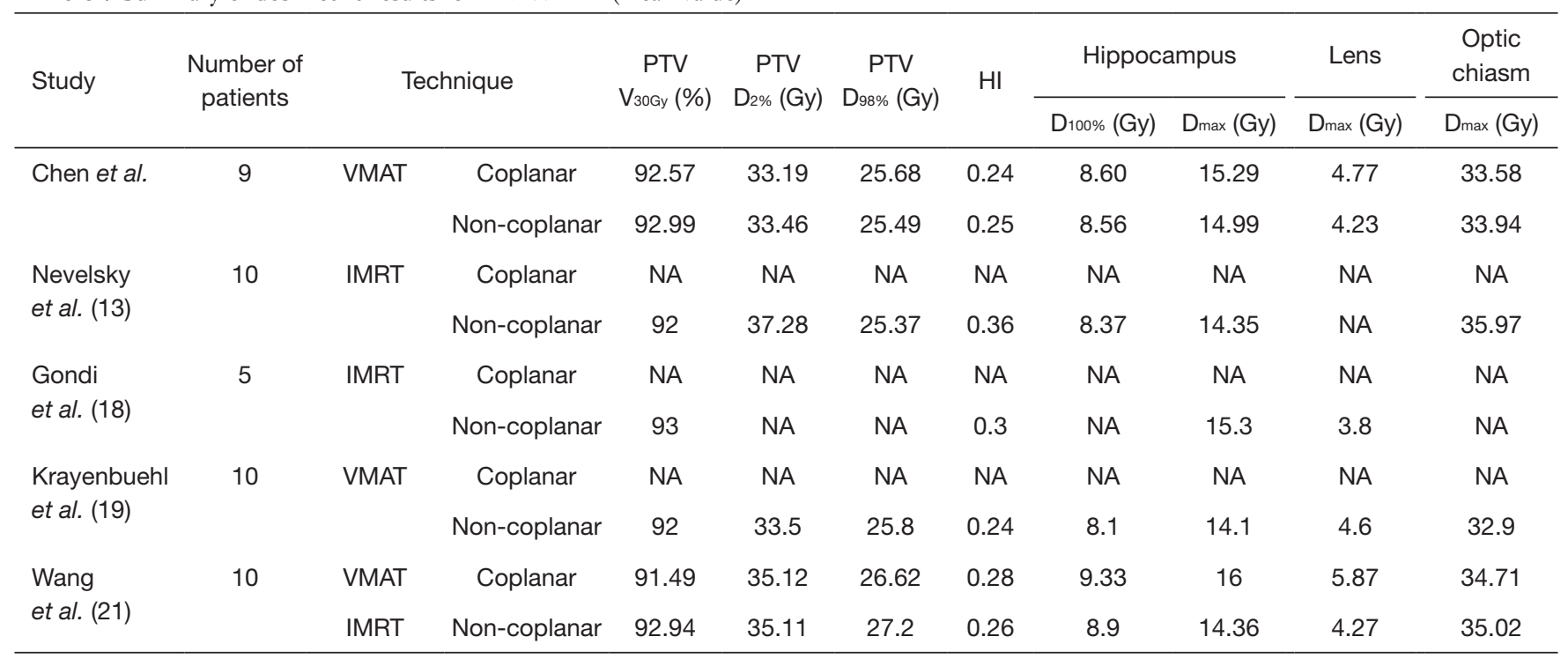

PTV, planning target volume; $\mathrm{V}_{30 \mathrm{G}}$, percent volume covered by $30 \mathrm{~Gy}$; $\mathrm{D}_{2 \%}$, maximum dose to $2 \%$ volume, D98\%, minimum dose to $98 \%$ volume; $\mathrm{D}_{100 \%}$, dose to $100 \%$ volume; Dmax, maximum dose; $\mathrm{HI}$, homogeneity index.

results showed that the average $\mathrm{D}_{\max }$ of the hippocampus was $15.3 \mathrm{~Gy}$, and the average $\mathrm{HI}$ was 0.3 . Our non-coplanar VMAT plans showed better average $\mathrm{D}_{\max }$ and $\mathrm{HI}$ values than those of Gondi et al. (18), and the shorter treatment time for the non-coplanar VMAT of this study can be expected due to less couch angle adjustment $\left(0^{\circ}\right.$ and $\left.70^{\circ}\right)$.

Krayenbuehl et al. (19) evaluated the feasibility of HAWBRT according to the RTOG 0933 trial using an automated TPS with VMAT of two FAs and two non-coplanar PAs. Their results showed that for 10 patients, the average $D_{\max }$ and minimum dose $\left(\mathrm{D}_{\min }\right)$ of the hippocampus were 14.1 Gy (range, 12-15.3 Gy) and 8.1 Gy (range, 7.8-8.5 Gy), respectively; the average PTV $V_{30 G y}$ and PTV $D_{\max }$ were $92 \%$ and 36 Gy (range, 35.1-36.5 Gy), respectively. In this study, we used the same TPS as the study performed by Krayenbuehl et al. (19), and our results for the non-coplanar VMAT plans showed that the average $\mathrm{D}_{\max }$ and $\mathrm{D}_{\min }$ of the hippocampus were $14.9 \mathrm{~Gy}$ (range, 13.8-15.8 Gy) and 8.5 Gy (range, 8.3-8.8 Gy). For the PTV, Krayenbuehl et al. (19) aimed to reduce the high dose in the normal brain (i.e., PTV $\mathrm{D}_{2 \%}$ ), and the average PTV $\mathrm{D}_{2 \%}$ of their study was $33.5 \mathrm{~Gy}$. In our study, the average PTV $\mathrm{D}_{2 \%}$ was $33.4 \mathrm{~Gy}$. Although our study showed a higher average PTV coverage (92.9\%) than that reported by Krayenbuehl et al. (19) $(92.0 \%)$, the $\mathrm{D}_{\max }$ and $\mathrm{D}_{\min }$ of the hippocampus in our study were both higher than their results but still met the criteria of the RTOG 0933 trial. Therefore, our results indicated that this study could reduce the high dose in the brain while maintaining PTV coverage. As for the better $D_{\max }$ and $D_{\min }$ of the hippocampus in the study of Krayenbuehl et al. (19), we believe that this can be explained by the usage of a Trilogy linac (Varian Medical System, Palo Alto, CA, USA) with 60 MLC pairs of $0.5 \mathrm{~cm}$ leaf width at isocenter. The narrower MLCs of the Trilogy compared with the MLCs of the Elekta Synergy used in this study can affect the treatment plan quality, since the width of the MLC would affect the spatial resolution of the dose distribution, and the penumbra width of the MLC could determine the dose fall-off in the hippocampal avoidance regions (20).

In the study performed by Wang et al. (21) using coplanar VMAT with two FAs, the average PTV $\mathrm{D}_{2 \%}$ and PTV $\mathrm{D}_{98 \%}$ were 35.1 and $26.6 \mathrm{~Gy}$, respectively, and the $\mathrm{D}_{\max }$ and $\mathrm{D}_{\text {min }}$ of the hippocampus were 16 and $9.3 \mathrm{~Gy}$, respectively. In this study, four FAs were used for the coplanar VMAT, which allowed us to achieve better PTV $\mathrm{D}_{2 \%}, \mathrm{PTV}_{98 \%}$, and $\mathrm{D}_{\max }$ and $D_{\min }$ values of the hippocampus than those reported by Wang et al. (21). Based on the results, we speculate that increasing the arc number of VMAT could be used to easily achieve the dose constraints of the RTOG 0933 trial but would increase the treatment time as well. The dosimetric comparison data from the studies mentioned above and our study are summarized in Table 7 .

For $\mathrm{D}_{\max }$ and $\mathrm{D}_{\min }$ of the hippocampus, our results indicated that no significant differences were observed 
between the non-coplanar and coplanar VMAT plans. For $\mathrm{D}_{\text {max }}$ of the lens, the non-coplanar VMAT plans showed a significantly lower value than the coplanar VMAT plans. Previously reported of radiation-induced lens change in animal and human radiation cataract in radiation workers, exposure of the lens to ionizing radiation can result in opacification (22). Nguyen et al. (23) proposed a mean lens dose threshold of 7 Gy, and they reported that the cataract risk can be reduced to less than $25 \%$. In addition, the National Council on Radiation Protection and Measurements No. 26 report suggests that it is more advantageous to keep the lens doses as low as possible according to the as low as reasonably achievable principle (24).

The non-coplanar technique could decrease the $\mathrm{D}_{\max }$ of the lens; however, due to the couch rotation during the treatment, the rotation accuracy for couch and gantry needs to be assessed before the non-coplanar treatment. The difference in treatment managements in clinical practice between the coplanar and non-coplanar VMAT plans results in the difference in delivery time. Although there was no statistically significant difference in the beamon time between the non-coplanar (average, $6.2 \mathrm{~min}$ ) and coplanar VMAT plans (average, $5.6 \mathrm{~min}$ ). In clinical practice, however, coplanar VMAT is performed with a fixed couch angle, and the couch angle needs to be adjusted for the non-coplanar VMAT. Therefore, we believe that the coplanar VMAT is less time-consuming than the noncoplanar VMAT. The shorter treatment time can reduce patient discomfort and decrease patient irritability during treatment.

Because VMAT involves continuous gantry rotation and leaf motion with a varying dose rate, it is important to verify the consistency of the TPS and beam delivery. In this study, we performed VMAT QA for each treatment plan to evaluate the feasibility of HA-WBRT. On average, the gamma passing rate with criteria of $3 \% / 3 \mathrm{~mm}$ was above $90 \%$, within the action level of $90 \%$ recommended by the AAPM Task Group 119 report (25). The profile shown in Figure 3 demonstrates that for the hippocampus, the measured dose distribution was in good agreement with the calculated plan dose distribution.

\section{Conclusions}

The application of VMAT for HA-WBRT has been a trend in clinical treatment for patients with brain metastases, and one advantage is the significant reduction in treatment time.
This study shows that the suggested criteria of the RTOG guidelines for the hippocampal dose can be achieved in both non-coplanar and coplanar VMAT plans. The non-coplanar VMAT plans showed a significantly lower $\mathrm{D}_{\max }$ of the lens than the coplanar VMAT plans. However, for the doses to other OARs, the coplanar VMAT plans showed comparable results. In addition, better treatment efficiency for coplanar VMAT than non-coplanar VMAT can be expected in clinical practice due to the shorter beam-on time and fixed couch angle. This study provides a dosimetric comparison of two different VMAT techniques for HA-WBRT, and we performed VMAT QA for each treatment plan to verify the clinical feasibility.

\section{Acknowledgments}

Funding: The authors disclosed receipt of the following financial support for the research and publication of this article: this work was supported by Taipei Medical University - Shuang-Ho Hospital (grant number: 107HCP-11).

\section{Footnote}

Reporting Checklist: The authors have completed the MDAR reporting checklist. Available at http://dx.doi.org/10.21037/ tro-20-50

Conflicts of Interest: All authors have completed the ICMJE uniform disclosure form (available at http://dx.doi. org/10.21037/tro-20-50). All authors report grants from Taipei Medical University - Shuang-Ho Hospital, outside the submitted work.

Ethical Statement: The authors are accountable for all aspects of the work in ensuring that questions related to the accuracy or integrity of any part of the work are appropriately investigated and resolved. The study was conducted in accordance with the Declaration of Helsinki (as revised in 2013). The study was approved by the Taipei Medical University Joint Institutional Review Board (TMU-JIRB No. N201711047), and informed consent was taken from all the patients

Open Access Statement: This is an Open Access article distributed in accordance with the Creative Commons Attribution-NonCommercial-NoDerivs 4.0 International License (CC BY-NC-ND 4.0), which permits the non- 
commercial replication and distribution of the article with the strict proviso that no changes or edits are made and the original work is properly cited (including links to both the formal publication through the relevant DOI and the license). See: https://creativecommons.org/licenses/by-nc-nd/4.0/.

\section{References}

1. Khuntia D, Brown P, Li J, et al. Whole-brain radiotherapy in the management of brain metastasis. J Clin Oncol 2006;24:1295-304.

2. Li J, Bentzen SM, Li J, et al. Relationship between neurocognitive function and quality of life after wholebrain radiotherapy in patients with brain metastasis. Int J Radiat Oncol Biol Phys 2008;71:64-70.

3. Chiesa S, Balducci M, Azario L, et al. Development of a modelling to correlate site and diameter of brain metastases with hippocampal sparing using volumetric modulated Arc therapy. Biomed Res Int 2013;2013:568597.

4. Kraft J, Zindler J, Minniti G, et al. Stereotactic Radiosurgery for Multiple Brain Metastases. Curr Treat Options Neurol 2019;21:6.

5. Sood S, Pokhrel D, McClinton C, et al. Volumetricmodulated arc therapy (VMAT) for whole brain radiotherapy: not only for hippocampal sparing, but also for reduction of dose to organs at risk. Med Dosim 2017;42:375-83.

6. Prado A, Milanés AI, Cabello E, et al. Dosimetric comparison of four volumetric-modulated arc therapy beam arrangements utilized for hippocampal-sparing whole-brain radiation therapy. J Med Phys 2019;44:1-8.

7. Rong Y, Evans J, Xu-Welliver M, et al. Dosimetric evaluation of intensity-modulated radiotherapy, volumetric modulated arc therapy, and helical tomotherapy for hippocampal-avoidance whole brain radiotherapy. PLoS One 2015;10:e0126222.

8. Warrington JP, Ashpole N, Csiszar A, et al. Whole brain radiation-induced vascular cognitive impairment: mechanisms and implications. J Vasc Res 2013;50:445-57.

9. Aoyama H, Shirato H, Tago M, et al. Stereotactic radiosurgery plus whole-brain radiation therapy vs stereotactic radiosurgery alone for treatment of brain metastases: a randomized controlled trial. JAMA 2006;295:2483-91.

10. De Felice F, Musio D, Cassese R, et al. Radiotherapeutic treatment approaches for brain metastases. Anticancer Res 2014;34:6913-8.
11. Mehta MP, Gondi V, Corn B, et al. A phase II trial of hippocampal avoidance during whole brain radiotherapy for brain metastases. RTOG 0933. Philadelphia, Pennsylvania: Radiation Therapy Oncology Group; 2011.

12. Ghia A, Tomé WA, Thomas S, et al. Distribution of brain metastases in relation to the hippocampus: implications for neurocognitive functional preservation. Int J Radiat Oncol Biol Phys 2007;68:971-7.

13. Nevelsky A, Ieumwananonthachai N, Kaidar-Person O, et al. Hippocampal-sparing whole-brain radiotherapy using Elekta equipment. J Appl Clin Med Phys 2013;14:4205.

14. Khan FM, Gibbons JP. Khan's the physics of radiation therapy. 5th ed. Philadelphia, Pennsylvania: Lippincott Williams and Wilkins; 2014.

15. Grégoire V, Mackie TR, De Neve W. Prescribing, recording, and reporting photon-beam intensitymodulated radiation therapy (IMRT): contents. J Int Comm Radiat Units Meas 2010;10:1-112.

16. Moon SY, Yoon M, Chung M, et al. Comparison of the extent of hippocampal sparing according to the tilt of a patient's head during WBRT using linear acceleratorbased IMRT and VMAT. Phys Med 2016;32:657-63.

17. Kumar SAS, George A. A study on the response of 2D ion chamber array detector for VMAT delivery. Int J Radiol Radiat Ther 2018;5:249-52.

18. Gondi V, Tolakanahalli R, Mehta MP, et al. Hippocampalsparing whole-brain radiotherapy: a "how-to" technique using helical tomotherapy and linear accelerator-based intensity-modulated radiotherapy. Int J Radiat Oncol Biol Phys 2010;78:1244-52.

19. Krayenbuehl J, Di Martino M, Guckenberger M, et al. Improved plan quality with automated radiotherapy planning for whole brain with hippocampus sparing: a comparison to the RTOG 0933 trial. Radiat Oncol 2017;12:161.

20. Huq MS, Das IJ, Steinberg T, et al. A dosimetric comparison of various multileaf collimators. Phys Med Biol 2002;47:N159-70.

21. Wang S, Zheng D, Zhang C, et al. Automatic planning on hippocampal avoidance whole-brain radiotherapy. Med Dosim 2017;42:63-8.

22. Kleiman NJ. Radiation cataract. Ann ICRP 2012;41:80-97.

23. Nguyen SM, Sison J, Jones M, et al. Lens dose-response prediction modeling and cataract incidence in patients with retinoblastoma after lens-sparing or wholeeye radiation therapy. Int J Radiat Oncol Biol Phys 2019;103:1143-50. 
24. Dauer LT, Ainsbury EA, Dynlacht J, et al. Guidance on radiation dose limits for the lens of the eye: overview of the recommendations in NCRP Commentary No. 26. Int J Radiat Biol 2017;93:1015-23.

doi: $10.21037 /$ tro-20-50

Cite this article as: Chen $\mathrm{LJ}, \mathrm{Li} \mathrm{MH}$, Cheng $\mathrm{HW}$, Kuo CY, Sun WL, Tsai JT. Hippocampus-sparing whole-brain radiotherapy: dosimetric comparison between non-coplanar and coplanar planning. Ther Radiol Oncol 2021;5:1.
25. Ezzell GA, Burmeister JW, Dogan N, et al. IMRT commissioning: multiple institution planning and dosimetry comparisons, a report from AAPM Task Group 119. Med Phys 2009;36:5359-73. 